PACIFIC JOURNAL OF MATHEMATICS

Vol. 179, No. 2, 1997

\title{
CHARACTERISTIC CLASSES FOR THE DEGENERATIONS OF TWO-PLANE FIELDS IN FOUR DIMENSIONS
}

\author{
Maxim Kazarian, Richard Montgomery and Boris Shapiro
}

Given a distribution of $k$-planes on a manifold, consider the degeneration locus $\Sigma_{I}$ consisting of points where the distribution has Lie-bracket growth vector less than or equal $I$, a fixed integer vector. We calculate the characteristic classes associated to the $\Sigma_{I}$ for a generic two-plane distribution on a four-manifold.

\section{Results and Background.}

\subsection{Generalities, Setting and Results.}

A distribution $\mathcal{D}$ of k-planes on an n-dimensional manifold $Q$ can be thought of as either a subbundle $\mathcal{D} \subset T Q$ of the tangent bundle or as a locally free sheaf of smooth vector fields. We use the same notation for both. Write $\mathcal{D}^{2}=\mathcal{D}+[\mathcal{D}, \mathcal{D}]$ and more generally $\mathcal{D}^{j+1}=\mathcal{D}^{j}+\left[\mathcal{D}, \mathcal{D}^{j}\right]$. These are sheaves of modules of vector fields (over the ring of smooth functions). We are interested in distributions such that for $r$ large enough we obtain all vector fields by this procedure:

$$
\mathcal{D}^{r}=T
$$

where $T$ denotes the sheaf of all vector fields. These are called completely nonholonomic distributions. We thus have a filtration

$$
\mathcal{D} \subset \mathcal{D}^{2} \subset \ldots \subset \mathcal{D}^{r}=T
$$

by subsheaves of the sheaf of all vector fields. Write $\mathcal{D}^{j}(q) \subset T_{q} Q$ for the vector subspace obtained by evaluating all vector fields in $\mathcal{D}^{j}$ at the point $q \in Q$, and

$$
n_{j}(q)=\operatorname{dim}\left(\mathcal{D}^{j}(q)\right) .
$$

The first $r$ such that $\mathcal{D}^{r}(q)=T_{q} Q$ is called the degree of nonholonomy at $q$ and the nondecreasing list of dimensions

$$
I(q)=\left(n_{1}(q), n_{2}(q), \ldots, n_{r}(q)\right)
$$


is called the growth vector at $q$. If the $n_{j}(q)$ are constant in a neighborhood of $q$ then the $\mathcal{D}^{j}$ correspond to vector bundles of this rank (in this neighborhood) which we will also denote by $\mathcal{D}^{j}$. In this case, we say that $q$ is a regular point for the distribution. Otherwise, some of the ranks $n_{j}$ jump as we pass through $q$ and we have to think of the corresponding $\mathcal{D}^{j}$ as sheaves.

Example. A contact distribution has growth vector $(k, k+1)$ with $k$ even.

Definition. An Engel distribution is a rank two distribution on a 4-dimensional manifold whose growth vector is $(2,3,4)$ everywhere.

What makes Engel distributions remarkable is that they are topologically stable, and topologically stable distributions are quite rare, occuring only in dimensions $(k, n)=(1, n),(n-1, n)$ and $(2,4)$. The only stable regular distributions are the line fields, the contact fields, an odd-rank analogue of contact (sometimes called pseudo-contact) and the Engel distribution. (See the next section, for the definition of stability, and some details.)

If one slightly perturbs any given distribution of 2-plane fields on a 4manifold then it will become Engel on an open dense subset ([11], [4]). On the other hand (see Propositon 1 below) if an oriented 4-manifold admits an oriented Engel distribution, then that manifold is parallelizable. Thus there are topological obstructions to making the 2-plane field globally Engel. Our goal is to understand these obstructions.

A basic notion in this endeavour will be the degeneraion locus of a distribution. We will first need to establish a partial order for growth vectors. Declare that $J=\left(m_{1}, m_{2},, m_{s}=n\right) \leq I=\left(n_{1}, n_{2}, \ldots, n_{r}=n\right)$ if and only if $m_{i} \leq n_{i}$ for $i=1, \ldots, r$. Note that the two vectors may have a different number of components. For fixed $k=n_{1}$ and $n$ there is exactly one maximal growth vectors. Its components, except for possibly the last one $n_{r}=n$ are the dimensions of the subspaces of the standard grading of the free Lie algebra on $\mathrm{k}$ elements. For typical distributions the growth vector will be maximal at most points of the manifold.

Definition. The degeneration locus of a distribution $\mathcal{D}$ on a manifold $Q$ is the set of all points $\Sigma=\Sigma(\mathcal{D}) \subset Q$ whose growth vector is less than maximal. The degeneration locus of type $I$, denoted $\Sigma_{I}=\Sigma_{I}(\mathcal{D}) \subset Q$, is the subset of all points $q \in Q$ at which the growth vector is less than or equal $I$.

The Thom transversality theorem implies that for typical $\mathcal{D}$ all of the $\Sigma_{I}$ are nice subvarieties. They stratify the manifold.

The Engel growth vector $(2,3,4)$ is the maximal growth vector for a rank two distribution in 4-space. The smaller growth vectors $I_{1}=(2,2,4), I_{2}=$ $(2,3,3,4)$ are not ordered relative to each other. All other growth vectors 
are dominated by these two. Consequently:

$$
\Sigma=\Sigma_{1} \cup \Sigma_{2}
$$

The following alternative descriptions of the $\Sigma_{i}$ may be more transparent:

$$
\begin{aligned}
& \Sigma_{1}=\left\{q: \operatorname{dim}\left(\mathcal{D}^{2}(q)\right) \leq 2\right\} \\
& \Sigma_{2}=\left\{q: \operatorname{dim}\left(\mathcal{D}^{3}(q)\right) \leq 3\right\} .
\end{aligned}
$$

Zhitomirskii has shown that generically each piece $\Sigma_{i}$ is a smooth 2-dimensional surface. Our question becomes, what is the topological meaning of the degeneration locus $\Sigma$ and its pieces $\Sigma_{1}$ and $\Sigma_{2}$ ? The situation is complicated by the fact that when the two pieces do intersect, they never do so transversally, but rather along a curve, denoted by $\mathrm{C}$ below.

In general, the condition that the growth vector $I(q)$ is less than or equal to some $I=\left(n_{1}, \ldots, n_{r}\right)$ defines a natural Diff $Q_{Q}$-invariant subset $\mathcal{M}_{\mathcal{I}}$ in the space of $r$-jets of sections of the bundle $G_{k, n}(T Q) \rightarrow Q$ whose fibers $G_{k, n}\left(T_{q} Q\right)$ are the Grassmannians of k-planes in the n-dimensional tangent spaces $T_{q} Q$, and where Diff ${ }_{Q}$ denotes the group of diffeomorphisms of $Q . \Sigma_{I}(\mathcal{D})$ is the pullback of the intersection of the $r$-jet extension of $\mathcal{D}$ with $\mathcal{M}_{\mathcal{I}}$. When we say 'typical' and 'generic' for $\mathcal{D}$, this means that the $r$-jet extension of $\mathcal{D}$ is transversal to the $\mathcal{M}_{\mathcal{I}}$. By results of Thom, (see [5]) for a generic $\mathcal{D}$ there exists a universal formula for the Stiefel-Whitney and, in the appropriate setting, the Chern classes which are Poincare dual to $\Sigma_{I}(\mathcal{D})$ in terms of the characteristic classes of $\mathcal{D}$ and $Q$. In this paper we obtain this formula for the Engel degenerations. The main result of this note is as follows.

Theorem 1. Let $\mathcal{D}$ be a real oriented 2-plane field on a closed oriented 4-manifold $Q$. Then the obstructions to $\mathcal{D}$ being Engel are the Chern classes $c_{1}\left(\mathcal{D}^{\perp}\right)$ and $c_{1}\left(\mathcal{D}^{*}\right)$. Here $\mathcal{D}^{\perp}=T Q / \mathcal{D}$ and $\mathcal{D}^{*}$ denotes the dual bundle. For generic $\mathcal{D}$ the first class is represented by $\Sigma_{1} \subset \Sigma$ which is a smooth 2-surface (except for possibly a finite number of points) with a global co-orientation. The second class is represented by $\Sigma$ with a certain co-orientation on $\Sigma \backslash C$ where $C=\Sigma_{1} \cap \Sigma_{2}$. The 1-cycle $C$ is homologous to zero in $\Sigma_{1}$.

Remark. $\quad \Sigma_{1}$ is canonically co-oriented as the class representing $c_{1}\left(\mathcal{D}^{\perp}\right)$. In fact it is the zero locus of a section of $\mathcal{D}^{\perp}$ which is transverse to the zero section. The co-orientation which $\Sigma_{1}$ receives as part of the cocycle $\Sigma$ representing $c_{1}\left(\mathcal{D}^{*}\right)$ reverses as the curve $\mathrm{C}$ is crossed. See Figure 1 below.

If the distribution $\mathcal{D}$ or the underlying manifold $Q$ are not oriented then the degeneration loci will not yield well-define integer homology classes, but 
rather only classes mod 2 . Let $w_{i}(\xi)$ denote the ith Steiffel-Whitney class of a real vector bundle, and write $w_{i}(Q)=w_{i}(T Q)$.

Theorem 2. The $\mathbb{Z}_{2}$-homology classes of $\Sigma_{1}, \Sigma_{2}, \Sigma_{1} \cup \Sigma_{2}$ and $\Sigma_{1} \cap \Sigma_{2}$ are dual to $w_{1}^{2}(D)+w_{2}(D)+w_{2}(Q), w_{1}^{2}(D)+w_{1}(Q) w_{1}(D)+w_{1}^{2}(Q)+w_{2}(Q)$, $w_{2}(D)+w_{1}^{2}(Q)+w_{1}(D) w_{1}(Q)$ and $w_{1}(D)\left(w_{1}^{2}(D)+w_{2}(D)+w_{2}(Q)\right)$ respectively.

Two of us (M. Kazarian and B. Shapiro), have found corresponding duality formulae for the case of generic distributions of arbitrary rank $\mathrm{k}$ in $\mathrm{n}$ dimensions, and plan to publish this in a future paper.

\subsection{Acknowledgements.}

We would like to thank Ivan Kupka for a careful reading and critique of an earlier version of this paper. R. Montgomery would like to thank the NSF, grant number DMS 9400515 and the University of California at Santa Cruz, FRC grants for support. M. Kazarian would like to thank the Russian Foundation for Basic Researches (RFSB) grant 95-01-01122a for support.

\subsection{Properties of rank two distributions on 4-manifolds.}

We formulate some known properties of rank two distributions $\mathcal{D}$ on a 4-manifold $Q$.

An oriented 4-manifold $Q$ admits an oriented 2-plane distribution $\mathcal{D}$ if and only if $\chi[Q] \equiv 0 \bmod 2$ and $\chi[Q]=\tau[Q] \bmod 4$. Here $\chi$ is the Euler class and $\tau$ the signature. This condition is equivalent to requiring that the manifold admit two almost complex structures, one consistent with the given orientation, and the other consistent with the opposite orientation. (See [2] and also [7] and references therein.)

If the distribution $\mathcal{D}$ has rank two then it is locally spanned by two nonvanishing vector fields $X$ and $W$. Locally:

$$
\mathcal{D}^{2}=\operatorname{span}\{X, W,[X, W]\}
$$

and

$$
\mathcal{D}^{3}=\operatorname{span}\{X, W,[X, W],[X,[X, W]],[W,[X, W]]\} .
$$

It follows that $\mathcal{D}$ is Engel if and only if for some functions $a, b$ the vector fields $X, W,[X, W]$ and $a[X,[X, W]]+b[W,[X, W]]$ form a basis for the tangent space.

Engel's Theorem. (See [10], [3, p. 50].) If $\mathcal{D}$ is Engel at the point $q \in Q$ then it admits a local frame $X$ and $W$ and $Q$ admits coordinates $(x, y, z, w)$ centered at $q$ such that $W=\frac{\partial}{\partial w}$ and $X=\frac{\partial}{\partial x}+w \frac{\partial}{\partial y}+y \frac{\partial}{\partial z}$. 
To clarify the special nature of Engel distributions, we recall the notion of "stability" in singularity theory. A distribution germ is called stable if every sufficiently nearby distribution germ is diffeomorphic to it, sufficiently near being measured in the $C^{r}$-topology for some $r$.

Stability Theorem. (See [10] and also [6].) The only stable distribution germs occur in dimensions $(k, n)=(1, n),(n-1, n)$ or $(2,4)$. In each case there is a unique stable regular representative. These are the line fields, contact (or even-contact) fields, and the Engel distributions.

The generic degenerations of Engel structures were classified, up to codimension 3, by Zhitomirskii [11]. The first degeneracies occur in codimension 2. There are two of them, one for $\Sigma_{1}$, and one for $\Sigma_{2}$. Both are stable. Corresponding normal forms were found by Zhitomirskii and are repeated below. The main result of Zhitomirskii's which we will be using is that for a Whitney open and dense set of rank two distributions on a 4-manifold, the degeneration loci $\Sigma_{1}$ and $\Sigma_{2}$ are smooth 2-dimensional embedded surfaces. Outside the intersection of $\Sigma_{1}$ and $\Sigma_{2}$, this follows from Thom's transversality theorem ([1, p. 38], and references therein).

We will also require the normal forms and higher singularities however. There are two types of codimension 3 degenerations. One occurs along $C=$ $\Sigma_{1} \cap \Sigma_{2}$ which is typically a curve when nonempty. The other occurs along a curve L in $\Sigma_{2}$ which separates the degenerating Engel line field near $\Sigma_{2}$ into hyperbolic and elliptic degenerations. The corresponding normal forms are given in terms of 1 -forms $\omega_{1}, \omega_{2}$ which frame the forms annihilating $\mathcal{D}$. They are

$$
\begin{aligned}
& \Sigma_{1}: \omega_{1}=d x_{1}+x_{3}^{2} d x_{4}, \omega_{2}=d x_{3}+x_{3} x_{4} d x_{4} \\
& \Sigma_{2}: \omega_{1}=d x_{1}+x_{3} d x_{4}, \omega_{2}=d x_{3}+\frac{1}{3}\left(x_{3}^{2}+x_{3} x_{4}\right) d x_{4}
\end{aligned}
$$

or

$$
\Sigma_{2}: \omega_{1}=d x_{1}+x_{3} d x_{4}, \omega_{2}=d x_{3}+x_{3}^{2} x_{4} d x_{4} .
$$

The two normal forms for $\Sigma_{2}$ correspond to the two regions of $\Sigma_{2}$ separated by the curve $\mathrm{L}$. For the points of $\mathrm{C}$ and $\mathrm{L}$ there is a single functional modulus f. Their normal forms are:

$$
\begin{aligned}
& C: \omega_{1}=d x_{1}+x_{3}^{2} d x_{4}, \omega_{2}=d x_{2}+x_{3}\left(x_{1}+f\right) d x_{4} \\
& L: \omega_{1}=d x_{1}+x_{3} d x_{4}, \omega_{2}=d x_{2}+x_{3} f d x_{4} .
\end{aligned}
$$

There are also codimension 4 singularities which occur along the curves $\mathrm{C}$ and L as isolated points. Fortunately we will not need explicit normal forms for them. (None have been calculated!) 
We re-iterate the main picture. $\Sigma$ is a smooth 2-dimensional surface away from the curve $\mathrm{C}$. Near $\mathrm{C}$ it is diffeomorphic to the interstion of two 2-planes in 4 -space which intersect on a line. See Figure 1 below.

\subsection{The Engel line field and parallelizability.}

We recall why Engel manifolds are parallelizable, up to orientation problems.

It will help to recall the basic Lie algebra structure associated to a distribution. Let [,] denote the operation of Lie bracket of vector fields. If $f$ and $g$ are smooth functions on an open set $U \subset Q$ and $X \in \mathcal{D}^{j}(U), Y \in \mathcal{D}^{k}(U)$ then

$$
[f X, g Y]=f g[X, Y] \bmod \left(\mathcal{D}^{l}\right)(U)
$$

where $l=\max (j, k)$. In other words, Lie bracket induces well-defined maps $\mathcal{D}^{j} \otimes \mathcal{D}^{k} \rightarrow T / \mathcal{D}^{l}$ where $T$ denotes the (sheaf of the) tangent bundle.

Associate to our filtration $\mathcal{D} \subset \mathcal{D}^{2} \subset \ldots \subset T$ the corresponding graded object:

$$
\operatorname{Gr}(T):=\mathcal{D} \oplus V_{2} \oplus V_{3} \oplus \ldots \oplus V_{r}
$$

where the

$$
V_{i}=\mathcal{D}^{i} / \mathcal{D}^{i-1}
$$

are the quotient sheaves. According to the remarks above, $\operatorname{Gr}(T)$ inherits the structure of a sheaf of graded nilpotent Lie algebras. Here "graded" means that

$$
\left[V_{i}, V_{j}\right] \subset V_{i+j}
$$

If the point $q$ is regular for $\mathcal{D}$ then the dimensions $n_{i}(q)$ are constant near $q$ so that the sheaves $\mathcal{D}^{i}$ correspond to smooth subbundles of the tangent space. In this case $\operatorname{Gr}(T)$ corresponds to a bundle of Lie algebras. (The simply connected Lie group corresponding to a particular fiber $\operatorname{Gr}\left(T_{q}\right)$ is called the nilpotentization of $(\mathcal{D}, Q)$ at $q$.) In the Engel case we have

$$
\operatorname{Gr}(T)=\mathcal{D} \oplus V_{2} \oplus V_{3}
$$

where $V_{2}$ and $V_{3}$ are real line bundles.

Fix a nonzero element $\delta \in V_{2}(q)$. Then the Lie bracket defines a map $\mathcal{D}(q) \rightarrow V_{3}(q) \cong \mathbb{R}$, namely $v \rightarrow[v, \delta]$. The kernel of this map is intrinsically defined and forms a line in the plane $\mathcal{D}(q)$. Said differently, in a neighborhood of any point where the distribution $\mathcal{D}$ is Engel there is a distinguished line field

$$
L \subset \mathcal{D}
$$


characterized by the fact that

$$
\left[L, \mathcal{D}^{2}\right]=0\left(\bmod \mathcal{D}^{2}\right)
$$

We call $L$ the Engel line field. It is the span of the vector field $W$ of Engel's theorem above.

Proposition 1. (See [4].) If an oriented 4-manifold admits an oriented Engel structure $\mathcal{D}$ then the manifold is parallelizable.

Proof. At every point $q$ of such a 4-manifold $Q$ we have the complete flag $L(q) \subset \mathcal{D}(q) \subset \mathcal{D}^{2}(q) \subset T_{q} Q$ in the tangent bundle. If we show that this flag is canonically oriented then we will be done. (A parallelization $\left\{E_{1}, E_{2}, E_{3}, E_{4}\right\}$ is then be obtained by putting a Riemannian metric on $Q$ and hence on each element of the flag. Then take $E_{1}$ to be the positive unit vector spanning $L,\left\{E_{1}, E_{2}\right\}$ to be the positive orthonormal basis for $\mathcal{D}$, etc.)

Write $A \in \Lambda^{2} \mathcal{D}$ for the choice of orientation of $\mathcal{D}$. Locally $A=X \wedge Y$ for $X, Y$ nonvanishing sections of $\mathcal{D}$. Then

$$
\delta_{1}=[X, Y](\bmod \mathcal{D})
$$

is a well-defined section of $T / \mathcal{D}$ independent of the choices of representation $\mathrm{X}$ and $\mathrm{Y}$. By the assumption on the growth vector it is non-vanishing and its span is the real line bundle $V_{2}$. So $\delta_{1}$ defines an orientation on $V_{2}$. Observe that for any triple of linear spaces, $(S, T, T / S)$ with $S \subset T$, an orientation on any two spaces canonically determines an orientation on the third.

Using this observation we obtain an orientation on $\mathcal{D}^{2}$. Applying the observation again we obtain an orientation on $V_{3}$. Finally, consider the map $a d_{\delta}: \mathcal{D} \rightarrow V_{3}$ defined by bracketing with $\delta$. Its kernel is $L$ so that $a d_{\delta}$ induces an isomorphism $V_{3} \cong \mathcal{D} / L$ and so an orientation on $\mathcal{D} / L$. Applying the observation again, we finally obtain the orientation on $L$.

Example 1. If $Q$ is closed and simply connected then it does not admit any Engel structure. This is because its Euler class is nonzero, and hence it does not admit a single nonvanishing vector field.

Example 2. If a 4-manifold admits one Engel structure, then it typically admits a continuous family of inequivalent such structures. For if we perturb the given structure, then we perturb its Engel line field. But line fields on closed manifolds typically have continuous moduli. For specific examples of families of inequivalent Engel structures, see [4]. The Engel situation is to be contrasted with the case of contact structures on a three-manifold, where the moduli space of inequivalent structures is a discrete set. 


\section{Obstruction theory and proofs.}

Our proofs rely on the following basic fact from topology. (See Bott and Tu, for example.) If $E \rightarrow Q$ is a real oriented rank 2 vector bundle and if $s: Q \rightarrow E$ is a section of $\mathrm{E}$ which is transverse to the zero section $Z \subset E$ then the section's zero locus represents the first Chern class $c_{1}(E)$ of E. (Bott and $\mathrm{Tu}$ call this the Euler class.) Transversality implies that this zero locus $s^{-1}(Z)=\{q: s(q)=0\}$ is a canonically co-oriented smooth submanifold of $Q$. It represents a cohomology class $\left[s^{-1}(Z)\right]$ via intersection theory. The value of $\left[s^{-1}(Z)\right]$ on a 2 -cycle is obtained by picking a representative for the cycle, jiggling it until it is transverse to $s^{-1}(Z)$ and then counting intersections.

The section $\delta_{1}$ of Equation 1:

$$
q \mapsto[X, Y](q) \bmod \mathcal{D}_{q}
$$

is a well-defined section of $\mathcal{D}^{\perp}=T Q / D$ regardless of whether or not $\Sigma$ is empty. Its zero locus is precisely $\Sigma_{1}$. Once we have proved transversality we will have established

Lemma 1. Let $\mathcal{D}$ be a generic oriented rank 2 distribution on an oriented 4-manifold $Q$. Then $\Sigma_{1}$ is canonically co-oriented and represents the first Chern class $c_{1}\left(\mathcal{D}^{\perp}\right)$ of the oriented rank 2 real vector bundle $\mathcal{D}^{\perp}=T Q / D$.

Proof. It remains to prove transversality of $\delta_{1}$. Away from $C=\Sigma_{1} \cap \Sigma_{2}$ the full growth vector is $(2,2,4)$, meaning that $[X,[X, Y]]$ and $[Y,[X, Y]](\bmod$ $\mathcal{D})$ span $\mathcal{D}^{\perp}$. It follows that by differentiating $\delta_{1}$ in the $\mathcal{D}$-directions we obtain all of $\mathcal{D}^{\perp}$. In other words, $\delta_{1}$ is transverse to $Z$ at these points and we only need to differentiate along $\mathcal{D}$ to achieve transversality.

At generic points of $\Sigma_{1} \cap \Sigma_{2}$ we will need to differentiate in directions other than $\mathcal{D}$, but transversality still holds. It follows from Zhitomirskii's normal form along $\mathrm{C}((4)$ above) that

$$
X_{1}=\partial_{3}
$$

and

$$
X_{2}=\partial_{4}-x_{3}^{2} \partial_{1}-x_{3}\left(x_{1}+f\right) \partial_{2}
$$

frame $\mathcal{D}$ near points $\mathrm{p}$ of the intersection. Here $\mathrm{p}$ has coordinates $(0,0,0,0)$ and $\mathrm{f}$ is a function satisfying $f(0)=d f(0)=0$. We may assume that the orientation of $\mathcal{D}$ is given by $A=X_{1} \wedge X_{2}$ since a nonzero scalar factor will 
not affect transversality considerations. The fields $\partial_{1}$ and $\partial_{2}$ form a local frame for $\mathcal{D}^{\perp}$. Modulo $\mathcal{D}$ we have:

$$
\delta_{1}=-2 x_{3} \partial_{1}-\left(x_{1}+f+x_{3} \partial_{3}(f)\right) \partial_{2}
$$

and

$$
\Sigma_{1}=\left\{x_{3}=0=x_{1}+f\right\}
$$

near $\mathrm{p}$. One calculates at $\mathrm{p}=0: \partial_{3} \delta_{1}=-2 \partial_{1}, \partial_{1} \delta_{1}=-\partial_{2}, \bmod \mathcal{D}$. Since $\partial_{1}, \partial_{2} \operatorname{span} \mathcal{D}$ this proves transversality.

Finally, we need to worry about the codimension 4 points. These form a finite set, some of which may lie along the curve $\Sigma_{1} \cap \Sigma_{2} . \Sigma_{1}$ may have singularities at these points. But these points do not effect cohomology. If a representative of a 2 -cycle intersects such a codimension 4 point it can be homotoped to miss it. And the co-orientation is defined everywhere on $\Sigma_{1}$ away from these points.

To obtain the other class, define a section $\delta_{2}$ of the vector bundle $\operatorname{Hom}\left(\mathcal{D}, \Lambda^{2} \mathcal{D}^{\perp}\right)$ by

$$
\delta_{2}(q)(v)=\delta_{1}(q) \wedge\left(\left[\tilde{v}, \tilde{\delta}_{1}\right](q)\left(\bmod \mathcal{D}_{q}\right)\right) .
$$

Here $v \in \mathcal{D}_{q}$ and $\tilde{v}$ denotes any extension of $\mathrm{v}$ to a local section of $\mathcal{D}$ : $\tilde{v}(q)=v$. And $\tilde{\delta}_{1}$ is any local vector field with the property that $\tilde{\delta}_{1}=$ $\delta_{1}(\bmod \mathcal{D})$. One easily checks that $\delta_{2}(q)(v)$ is well-defined, independent of these choices. It is clear that $\mathcal{D}$ fails to be Engel exactly at those points $q$ for which $\delta_{2}(q)(v)=0$ for all $\mathrm{v}$, that is to say at $\delta_{2}^{-1}(0)$. Thus

$$
\Sigma:=\Sigma_{1} \cup \Sigma_{2}=\delta_{2}^{-1}(0)
$$

$\mathcal{D}$ and $T Q$ are oriented, so that $\Lambda^{2} \mathcal{D}^{\perp}$ is trivial and $\operatorname{Hom}\left(\mathcal{D}, \Lambda^{2} \mathcal{D}^{\perp}\right) \cong \mathcal{D}^{*}$. Thus $\delta_{2}$ defines a section of $\mathcal{D}^{*}$. If this section were transverse to the zero section we would be done, as described at the beginning of this section. However the section $\delta_{2}$ cannot be transverse to the zero section at points of $C=\Sigma_{1} \cap \Sigma_{2}$ because $\Sigma$ is not a manifold at such points. In this case further analysis is needed and this complicates the proof of Theorem 1 . The theorem follows from

Lemma 2. For a generic distribution $\mathcal{D}$ the section $\delta_{2}$ is transverse to the zero section away from the curve $C=\Sigma_{1} \cap \Sigma_{2}$. The $\delta_{2}$-induced coorientations on either $\Sigma_{i}, i=1,2$ reverses as $C$ is crossed. See Figure 1. $C$ is homologous to zero within $\Sigma_{1}$ but might not be homologous to zero 
within $\Sigma_{2} . \Sigma$, being a Whitney stratified set with "cohomologically consistent co-orientations" ( see discussion below) on its principal strata, defines a cohomology class. This class represents the first Chern class $c_{1}\left(\mathcal{D}^{*}\right)$ of the dual to $\mathcal{D}$ bundle $\mathcal{D}^{*}$.

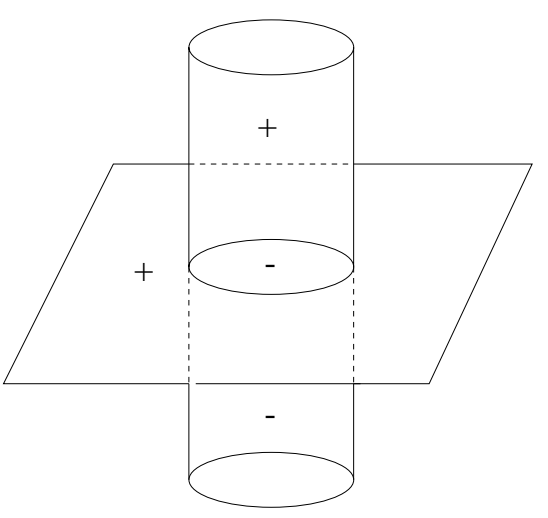

a) local structure of the zero locus

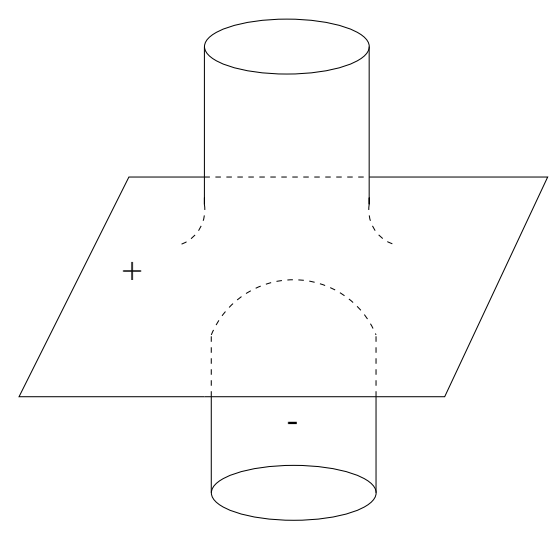

b) the zero locus after the perturbation

Figure 1. Local coorientation of $\Sigma=\Sigma_{1} \cup \Sigma_{2}$.

We postpone the proof of the lemma for a discussion of the business of Whitney stratified sets defining cohomology classes. It follows from Zhitomirskii's normal forms (Eq. (1), (2), (3), (4), (5)) that $\Sigma$ is a Whitney stratified subset of $Q$ of a rather tame sort. Its strata are the isolated codimension 4 points $\left\{p_{1}, \ldots, p_{N}\right\}$ (their normal forms have functional moduli and are not given by Zhitomirskii), the curve $C$ minus points of codimension 4 and the connected components of $\Sigma \backslash C$. These latter are the principal strata. They intersect along $\mathrm{C}$ in a manner locally diffeomorphic to that of two 2-planes in 4-space intersecting along a line.

We now describe how co-oriented Whitney stratified sets may be used to define cohomology classes. This idea is extensively used by Vassiliev and we refer the reader to his book [9], especially the introduction and $§ 8.4$. Let $Q$ be a smooth compact manifold and $W \subset Q$ be a Whitney stratified compact subset. Suppose that the principal strata of $\mathrm{W}$ are co-oriented kdimensional submanifolds. Given an $(n-k)$-cycle in $\mathrm{Q}$ we perturb it slightly so that it intersects the principal strata of $\mathrm{W}$ transversally and then count these intersection points with a plus or minus sign depending on whether the orientation of the cycle there agrees or disagrees with the co-orientation. In this way it appears that $\mathrm{W}$ yields an integer-valued function on cycles. However this number may depend on the perturbation and may not be welldefined on the level of homology. In other words, we must somehow insure 
that if a cycle $\mathrm{Z}$ is a boundary then this intersection number is zero. By cellular or simplicial approximation we may break $\mathrm{Z}$, and also the $(n-k+1)$ cycle B which it bounds into a sum of cycles each one of which is supported in an arbitrarily small neighborhood of Q. First suppose the intersection $B \cap W$ lies entirely on a principal stratum. Since we may assume that it is arbitrarily small, this intersection is diffeomorphic to an oriented line segment and $Z \cap W$ consists of two points with opposite orientations. Consequently its intersection numbers add to zero as desired. If $B$ intersects a stratum $\mathrm{R}$ of dimension $k-1$ we must impose a cohomological consistency condition, to be spelled out momentarily, on $\mathrm{W}$ near $\mathrm{R}$. If $\mathrm{B}$ intersects a stratum of dimension $k-2$ or less then we may slightly perturb it while keeping its boundary fixed, and in this way reduce to the case of intersection with a strata of dimension $k$ or $k-1$.

Cohomological Consistency Condition: The link L of any stratum of dimension $k-1$ is cohomologically trivial within the sphere of dimension $n-k$. See Figure 2 .

This link is a finite collection of co-oriented points on the $(n-k)$-sphere. The condition is then that the sum of co-orientations is zero. In particular the number of points in the link must be even.

OUR CASE: See the right hand picture of Figure 2. In our case $k=2$ so $k-1=1$. The 1 -dimensional stratum consists of the smooth points of $\mathrm{C}$, that is all the points of $\mathrm{C}$ except the points of codimension 4 not covered in Zhitomirskii's paper. The link of $\mathrm{C}$ consists of 4 points, representing the 4 'pieces' of $\Sigma \backslash C$ near C. The cohomological condition is that two of these points have plus signs and two have minus signs.
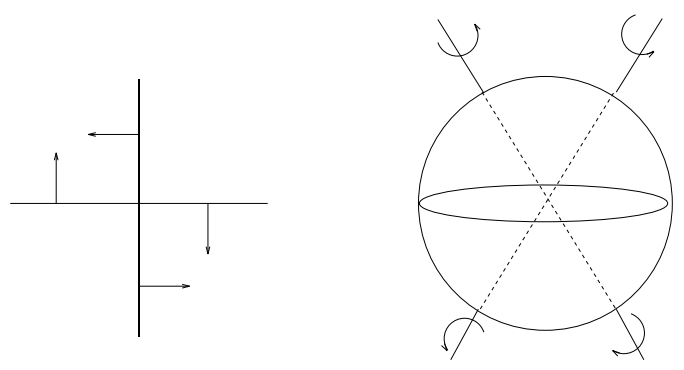

Figure 2. Local coorientations for the case $n-k=1$ and $n-k=2$.

We recall that the notion of the link $\mathrm{L}$ of a singular stratum $\mathrm{R}$. $\mathrm{R}$ is a piece of a submanifold of dimension $\mathrm{r}$, where $r \leq k-1$. Intersect $\mathrm{R}$ transversally at a point $\mathrm{p}$ with a small piece of an $(n-r)$-manifold $\mathrm{V}$. Intersect the result with an $(n-r-1)$-sphere $S \subset V$ surrounding p. We call L, or the pair $(L, S)$ the LINK of $\mathrm{R}$ at p. By the definition of a stratified set, $V \cap W$ is 
diffeomorphic to the cone $C(L)$ over $\mathrm{L}$, and a p admits a neighborhood $\mathrm{N}$ and a diffeomorphism which takes the pair $(W \cap N, N)$ to $(U \times C(L), U \times V)$ for some open set $\mathrm{U}$ in $\mathrm{R}$.

Lemma 3. The cohomological condition insures that $W$ is a well-defined cohomology class.

Proof. It remains to show that if the small $(n-k+1)$-cycle B intersects a stratum of dimension $k-1$ then the intersection number of its boundary $\mathrm{Z}$ with $\mathrm{W}$ is zero. We may take the cycle $\mathrm{B}$ to be ball intersecting $\mathrm{R}$ transversally. Its boundary can be homotoped so as to form the sphere $\mathrm{S}$ used to define the link $\mathrm{L}$ of $\mathrm{R}$. Then the intersection $Z \cap W$ is the sum of the points $\mathrm{L}$ with appropriate intersection numbers, which we have assumed to be zero.

Proof of Lemma 2. If $\delta_{2}$ is transverse to the zero section away from $\mathrm{C}$ as claimed, and if the induced co-orientations on $\Sigma \backslash C$ reverse as $\mathrm{C}$ is crossed while travelling along a fixed $\Sigma_{i}$, then the cohomological consistency condition follows directly. For the link consists of four points and this reversal implies they come in pairs which cancel, one pair for each $\Sigma_{i}$. See Figure 2.

The claim regarding the Chern class also follows directly. To see this imagine a cycle $\mathrm{K}$ and its intersection number with $\Sigma$ as we have just defined it. The intersection points with $\mathrm{K}$ lie outside some small neighborhood $\mathrm{U}$ of C. By Sard's theorem, we may perturb the section $\delta_{2}$ so that the resulting section $\mathrm{s}$ is transverse to the zero section $\mathrm{Z}$ of $\mathcal{D}^{*}$. Moreover, this perturbation may be concentrated within $\mathrm{U}$ so that $\mathrm{s}$ agrees with $\delta_{2}$ outside of $\mathrm{U}$. Then the intersection number of $\mathrm{K}$ with $\Sigma$ equals that of $\mathrm{K}$ with $s^{-1}(0)$. The later represents the first Chern class of $\mathcal{D}^{*}$ since $\mathrm{s}$ is a transverse section.

\section{See Figure 1 again.}

It remains to check the transversality claims. We will only check them near C. For points of $\Sigma$ away from C or L transverality follows from the normal forms (1), (2), (3) given above. The calculation follows the lines we follow below, but are significantly simpler. For points along $\mathrm{L}$ the calculation is similar to that below and is also omitted. We would use the form (5).

For points along $\mathrm{C}$ we use (1) in the dual form of equations (7) - (10) which follow Lemma 1. We have

$$
\left[X_{1}, \delta_{1}\right]=-2 \partial_{1}-\left(2 \partial_{3} f+x_{3} \partial_{3}^{2} f\right) \partial_{2}
$$

and

$$
\left[X_{2}, \delta_{2}\right]=\left\{X_{2}\left[f+x_{3} \partial_{3} f\right]-\delta_{1}\left[x_{3}\left(x_{1}+f\right)\right]\right\} \partial_{2}=\left\{\partial_{4} f+O(2)\right\} \partial_{2}
$$


If $x \in \Sigma_{1}$ then $\delta_{1}$ must be proportional to $\left[X_{1}, \delta_{1}\right]$. Multiplying our expression for $\left[X_{1}, \delta_{1}\right]$ by $2 x_{3}$ and refering back to Equation (9) for $\delta_{1}$ we see that this means that:

$$
x_{1}+f=x_{3} h
$$

where $\mathrm{h}$ is a certain function vanishing to 1st order. Also we must have $\left[X_{2}, \delta_{1}\right]=0$ when $x_{3} \neq 0$ since in this case $\delta_{1}$ has a $\partial_{1}$-component whereas $\left[X_{2}, \delta_{1}\right]$ has only a $\partial_{2}$-component. Now we may assume, by genericity, that $d\left(\partial_{4} f\right) \neq 0$ so that the leading term of this $\partial_{2}$-component is $\partial_{4} f$. We may then write $\left[X_{2}, \delta_{2}\right]=\bar{x}_{4} \partial_{2}$ where $\bar{x}_{4}=\partial_{4} f+O(2)$ is a new coordinate. Also set

$$
\tilde{x}_{1}=x_{1}+f .
$$

Generically we have $d \tilde{x}_{1} \wedge d x_{2} \wedge d x_{3} \wedge d \bar{x}_{4} \neq 0$ so that $\left(\tilde{x}_{1}, x_{2}, x_{3}, \bar{x}_{4}\right)$ form coordinates near p. Observe that $\Sigma_{1}$ can equally well be expressed as the locus of points with $x_{3}=0$ and $\tilde{x}_{1}-x_{3} h=0$. Set

$$
\bar{x}_{1}=\tilde{x}_{1}-x_{3} h .
$$

Thus locally

$$
\begin{aligned}
& \Sigma_{1}=\left\{x_{3}=0, \bar{x}_{1}=0\right\}, \\
& \Sigma_{2}=\left\{\bar{x}_{1}=0, \bar{x}_{4}=0\right\},
\end{aligned}
$$

and

$$
C=\left\{x_{3}=0, \bar{x}_{1}=0, \bar{x}_{4}=0\right\} .
$$

Note that this expresses the intersection $\mathrm{C}$ as a line obtained by intersecting two 2-planes in 4-space as claimed.

The section $\delta_{2}$ is a fiber-linear map which is given on the basis $\left\{X_{1}, X_{2}\right\}$ by $X_{i} \mapsto \delta_{1} \wedge\left[X_{i}, \delta_{1}\right]$. Using the above formulae we compute that we can represent $\delta_{2}$ as the 2 -vector:

$$
\delta_{2}=\left(-2 \bar{x}_{1}+x_{3} g,-2 x_{3} \bar{x}_{4}\right)
$$

where $\mathrm{g}$ is function vanishing to 1 st order at $\mathrm{p}$. We have also dropped off the basis vector coefficient $\partial_{1} \wedge \partial_{2}$ from $\delta_{2}$. (Here $(1,0),(0,1)$ represent the dual basis to $X_{1}, X_{2}$.) Then near $\mathrm{p}$ we have:

$$
\begin{aligned}
\frac{\partial}{\partial \bar{x}_{1}} \delta_{2} & =(-2,0), \\
\frac{\partial}{\partial x_{3}} \delta_{2} & =\left(g,-2 x_{4}\right) .
\end{aligned}
$$


If $X_{1} \wedge X_{2}$ represents the orientation of $\mathcal{D}$ then $\{(1,0),(0,1)\}$ represent the corresponding oriented frame for $\operatorname{Hom}\left(\mathcal{D}, \Lambda^{2} \mathcal{D}\right)=\mathcal{D}^{*}$. Observe that the normal bundle to $\Sigma_{1}$ near $\mathrm{p}$ is framed by $\frac{\partial}{\partial \bar{x}_{1}}$ and $\frac{\partial}{\partial x_{3}}$. It follows from our expressions for the derivatives of $\delta_{2}$ that the $\delta_{2}$-induced co-orientation of $\Sigma_{1} \backslash C$ is given by the frame $\left\{\frac{\partial}{\partial \bar{x}_{1}}, \bar{x}_{4} \frac{\partial}{\partial x_{3}}\right\}$. The induced co-orientation reverses as we cross $C$ travelling on $\Sigma_{1}$, since $C$ is defined on $\Sigma_{1}$ by $\bar{x}_{4}=0$. Also, the section $\delta_{2}$ is transverse to the zero section for points of $\Sigma_{1}$ away from $\mathrm{C}$.

To complete the proof we compute:

$$
\frac{\partial}{\partial \bar{x}_{4}} \delta_{2}=\left(0,-2 x_{3}\right)
$$

The vector fields $\frac{\partial}{\partial \bar{x}_{1}}$ and $\frac{\partial}{\partial \bar{x}_{4}}$ frame the normal bundle to $\Sigma_{2}$ near p. For the same reasons as above we see that the $\delta_{2}$-induced co-orientation of $\Sigma_{2}$ is given by the frame $\left\{\frac{\partial}{\partial \bar{x}_{1}}, x_{3} \frac{\partial}{\partial x_{4}}\right\}$. On $\Sigma_{2}$, the curve $\mathrm{C}$ is given by $x_{3}=0$. Again the induced co-orientation reverses as we cross $C$ and the section is transverse away from $\mathrm{C}$.

Remark. The co-orientability of $\Sigma_{2}$ depends on the orientability of the 3 -distribution $\left.D^{3}\right|_{\Sigma_{2}}$. One can check that the subvariety in the 2-jets of germs of 2-distributions corresponding to the growth vector $(2,3,3)$ is not coorientable.

Proof of Theorem 2. The construction of the sections $\delta_{1}$ and $\delta_{2}$ can be adjusted to the nonoriented case to define sections of bundles $\operatorname{Hom}\left(\Lambda^{2} \mathcal{D}, \mathcal{D}^{\perp}\right)$ and $\operatorname{Hom}\left(\mathcal{D}, \Lambda^{2}\left(\mathcal{D}^{\perp}\right)\right)$ such that $\Sigma_{1}$ and $\Sigma=\Sigma_{1} \cup \Sigma_{2}$ are the zero loci of these sections. Therefore, these cycles are dual to the StiefelWhitney classes of the corresponding bundles which can be calculated by using standard methods of the theory of characteristic classes. In particular, we have,

$$
\begin{aligned}
{\left[\Sigma_{1}\right] } & =w_{2}\left(\operatorname{Hom}\left(\Lambda^{2} \mathcal{D}, \mathcal{D}^{\perp}\right)\right)=w_{1}^{2}(\mathcal{D})+w_{2}(\mathcal{D})+w_{2}(Q) ; \\
{\left[\Sigma_{1} \cup \Sigma_{2}\right] } & =w_{2}\left(\operatorname{Hom}\left(\mathcal{D}, \Lambda^{2}\left(\mathcal{D}^{\perp}\right)\right)=w_{2}(\mathcal{D})+w_{1}^{2}(Q)+w_{1}(\mathcal{D}) w_{1}(Q) ;\right. \\
{\left[\Sigma_{2}\right] } & =\left[\Sigma_{1}\right]+\left[\Sigma_{1} \cup \Sigma_{2}\right]=w_{1}^{2}(\mathcal{D})+w_{1}(Q) w_{1}(\mathcal{D})+w_{1}^{2}(Q)+w_{2}(Q) .
\end{aligned}
$$

If the restriction of $\mathcal{D}$ to $\Sigma_{1}$ is orientable then the arguments at the beginning of this section show that $\Sigma_{1} \cap \Sigma_{2}$ is a boundary. Therefore $\Sigma_{1} \cap \Sigma_{2}$ represents the homology class on $\Sigma_{1}$ dual to $w_{1}\left(\left.\mathcal{D}\right|_{\Sigma_{1}}\right.$ ). (Using a bundle homomorphism which is essentially given by $\delta_{2}$ one can explicitly find a bundle over $\Sigma_{2}$ isomorphic to $\left.\Lambda^{2} \mathcal{D}\right|_{\Sigma_{1}}$ and a section of it for which $\Sigma_{1} \cap \Sigma_{2}$ is the zero locus.) Hence, we can apply the Gysin formula to compute the cohomology 
class dual to $\Sigma_{1} \cap \Sigma_{2}$ as follows

$$
\left[\Sigma_{1} \cap \Sigma_{2}\right]=i_{*}\left(\left[\left.\Sigma_{1} \cap \Sigma_{2}\right|_{\Sigma_{1}}\right]\right)=i_{*}\left(w_{1}\left(\left.\mathcal{D}\right|_{\Sigma_{1}}\right)\right)=w_{1}(\mathcal{D}) i_{*}(1)=w_{1}(\mathcal{D})\left[\Sigma_{1}\right]
$$

Here $\left[\left.\Sigma_{1} \cap \Sigma_{2}\right|_{\Sigma_{1}}\right]$ denotes the class dual to the cycle $\Sigma_{1} \cap \Sigma_{2}$ in the cohomology group of $\Sigma_{1}, i: \Sigma_{1} \rightarrow Q$ is the natural inclusion and $i_{*}$ is the corresponding Gysin homomorphism in cohomology.

\section{Open problems.}

Problem 1. Does every closed parallelizable 4-manifold admit an Engel structure? (The proof suggested in [4] is incomplete.)

This problem, the converse to the parallelizability proposition, is the basic open question in the area. The vanishing of the obstructions $c_{1}\left(\mathcal{D}^{\perp}\right)$ and $c_{1}\left(\mathcal{D}^{*}\right)$ of our main theorem implies the parallelizability of $Q$. On the other hand, a parallelizable $Q$ typically admits countably many homotopically distinct 2 -distributions $\mathcal{D}$ satisfying these vanishing conditions. The next problem is a stronger version of Problem 1.

Problem 2. Suppose that the rank two distribution $\mathcal{D}$ satisfies $c_{1}\left(\mathcal{D}^{\perp}\right)=$ $c_{1}\left(\mathcal{D}^{*}\right)=0$. Can we homotope $\mathcal{D}$ to an Engel distribution?

\section{References}

[1] V.I. Arnold, S.M. Gusein-Zade and A.N. Varchenko Singularities of Differentiable Maps, Vol. 1, Birkhauser, Boston, 1985.

[2] M. Atiyah, Vector fields on manifolds, in "Collected Works", Vol. 2, (1988), 729747.

[3] R.L. Bryant, S.S. Chern, R.B. Gardner, H.L. Goldschmidt and P.A. Griffiths Exterior Differential Systems, Vol. 18, MSRI Publications, Springer-Verlag, 1991.

[4] V. Gershkovich, Engel Structures on 4-Dimensional Manifolds, preprint, U. of Melbourne, 1993.

[5] A.Haefliger and A.Kosinski, Une theoreme de Thom sur les singularités des applications différentiables, Semin. H. Cartan, E.N.S, exp. 8, 1956-1957.

[6] R. Montgomery, Generic distributions and Lie algebras of vector fields, J. of Diff. Eqs., 103(2) (1993), 387-393.

[7] Y.Matsushita, Fields of 2-planes on compact simply-connected smooth 4-manifolds, Math. Ann., 280 (1988), 687-689.

[8] I.R. Porteus, Simple singularities of maps, Lecture Notes in Math., 192 (1971), 286-312.

[9] V.A. Vassiliev, Lagrange and Legendre Characteristic Classes, Advanced Studies in Contemporary Mathematics series, Vol. 3, Gordon and Breach Sci. Pub., 1988. 
[10] A.M. Vershik and V. Ya Gerhskovich, Nonholonomic Dynamical Systems, Geometry of Distributions and Variational Problems, in Dynamical Systems VII ed. V.I. Arnol'd and S.P. Novikov, Vol. 16 of the Encyclopaedia of Mathematical Sciences series, Springer-Verlag, NY, 1994. (Russian original 1987.)

[11] M. Ya. Zhittomirskii, Normal Forms of germs of 2-dimensional distributions in $\mathbb{R}^{4}$ Funktsional. Anal. I Ego Prilozhen., 24(2), 81-82. English translation: Funct. Anal. Appl. 24, (1990).

Received October 15, 1995 and revised July 19, 1996.

Steklov Mathematical Institute

42 VAVIlova St., 117966

Moscow GSP-1, Russia

E-mail address: kazarian@ium.ips.ras.ru

UNIVERSITY OF CALIFORNIA

SANTA CRUZ, CA 95064

E-mail address: rmont@cats.ucsc.edu

AND

UNIVERSITY OF STOCKHOLM

STOCKHolm, S-10691

SWEDEN

E-mail address: shapiro@matematik.su.se 